IRSTI 34.15 .51

\author{
${ }^{1,2 *}$ S.K. Davletova \\ 'Present address: Department of Biology and Biotechnology, \\ al-Farabi Kazakh National University, Almaty, Kazakhstan \\ ${ }^{2}$ Department of Otolaryngology-Head and Neck Surgery, Ohio State University, Columbus, Ohio, USA. \\ *e-mail: davlets@gmail.com
}

\title{
Merlin tumor suppressor signaling in the neurofibromatosis type 2 tumorigenesis
}

\begin{abstract}
In this review a complex signaling in human neurofibromatosis type 2(NF2) in nervous system and other tissues is discussed. The neurofibromatosis type 2 (Nf2) gene, known as a tumor suppressor, encodes a protein Merlin, which is closely related to the ezrin, radixin, and moesin (ERM) proteins of the band 4.1 protein superfamily. Mutations of the NF2 gene cause an autosomal dominant disorder neurofibromatosis type 2 (NF2). Merlin's function as a growth suppressor depends on its structural conversion from an inactive to an active form caused by changes in its phosphorylation status. Depending on its structural conformation Merlin binds to numerous interacting proteins, which are either required for Merlin's tumour suppressor function or impair Merlin's growth suppressor activity contributing to tumorigenesis.

Despite significant progress made in the NF2 study for the last two decade, the molecular mechanisms of the tumor suppressor function of Merlin are still little understood. Loss of the $N f 2$ function leads to aberrant activation of receptor tyrosine kinases (RTK) and the downstream mitogenic signaling pathways. Overexpression of ErbB receptors has been reported in NF2-related and sporadic vestibular schwannomas (VS) and many other human cancers. Studies have shown that inhibition of the ErbB family RTKs can serve as an alternative approach for the treatment of sporadic and NF2-related VS through inhibition of ERK1/2 and PI3K/AKT signaling pathways. Preclinical and clinical studies of the potential therapeutic targets for the treatment of VS, and other brain tumors are underway. A better understanding of Merlin's deregulated signaling in NF2 can provide useful therapeutic strategies for treatment of human VS and other brain tug mors.
\end{abstract}

Key words: Neurofibromatosis type 2, NF2, Merlin, vestibular schwannoma, receptor tyrosine kinases.

\section{Introduction}

Neurofibromatosis type 2 (NF2) is bilateral acoustic neurofibromatosis, a dominantly inherited autosomal disorder associated with the development of nervous system tumors [1]. The Nf2 gene encodes for Merlin (for moesin, ezrin, radixin-like protein), a member of the band 4.1 superfamily of membraneassociated proteins [2]. Merlin is implicated in diverse processes at different stages of development and is critical for embryonic development of different cell types [3]. Loss of $N f 2$ function was lethal for embryonic mice, while heterozygous $N f 2$-mutant mice developed metastatic tumors [4]. The Nf2 disfunction in the liver resulted in an expansion of progenitor cells, suggesting that Merlin is involved in regulation of stem-cell renewal $[5 ; 6]$. Numberous studies have shown that Merlin functions as a negative regulator of cell growth and proliferation [7;8]. Mutation in the Nf2 leads to the development of the nervous system tumors, such as schwannomas, meningiomas, and ependymomas $[4 ; 9]$, as well as cancers of nonnervous system, mainly mesotheliomas [10], glioblastomas [11] and in rare cases to other solid tumors [12]. In addition, sporadic non-germline loss of $N F 2$ function has been observed nearly in all vestibular schwannomas, meningiomas, and to a lesser extends in ependymomas.

The band 4.1 superfamily proteins are cytoskeletal linker proteins, which are involved in the cytoskeleton organization. Merlin has high homology with the ERM (for ezrin, radixin, moesin) family proteins and shares a common structural organization: the global N-terminal FERM (fourpoint-one, ERM) domain (NTD), the central $\alpha$-helical domain and the C-terminal domain (CTD) (Figure 1). In contrast to other ERM family proteins, Merlin lacks the actinbinding motif at the CTD region. Despite the struc- 
tural similarities, Merlin is the only protein of this family, which possesses growth-suppressive activity. The Nf2 gene encodes two isoforms such as Merlin (or Merlin-1), which has the CTD, and Merlin-2, which lacks the CTD. The isoform 1 contains exon 17 and lacks exon 16, while the isoform 2 contains exon 16 , followed by a termination codon that prevents the translation of exon 17 . It has been generally accepted that the isoform 2 does not possess the tumor suppressor activity. Nevetheless, studies have shown that both isoforms possess the tumor suppres- sor activity [13]. Most of mutations within the FERM domain lead to cell proliferation and tumorigenesis. This indicates that the FERM domain in Merlin is crucial for contact inhibition and tumor suppression. It was believed that Merlin acquired tumor suppressive activity, by adopting a closed growth-suppressive conformation through the intramolecular association of its NTD and the CTD [14]. However, subsequent studies have revealed a more complex regulation of Merlin's growth suppressive function, which will be discussed in this review.

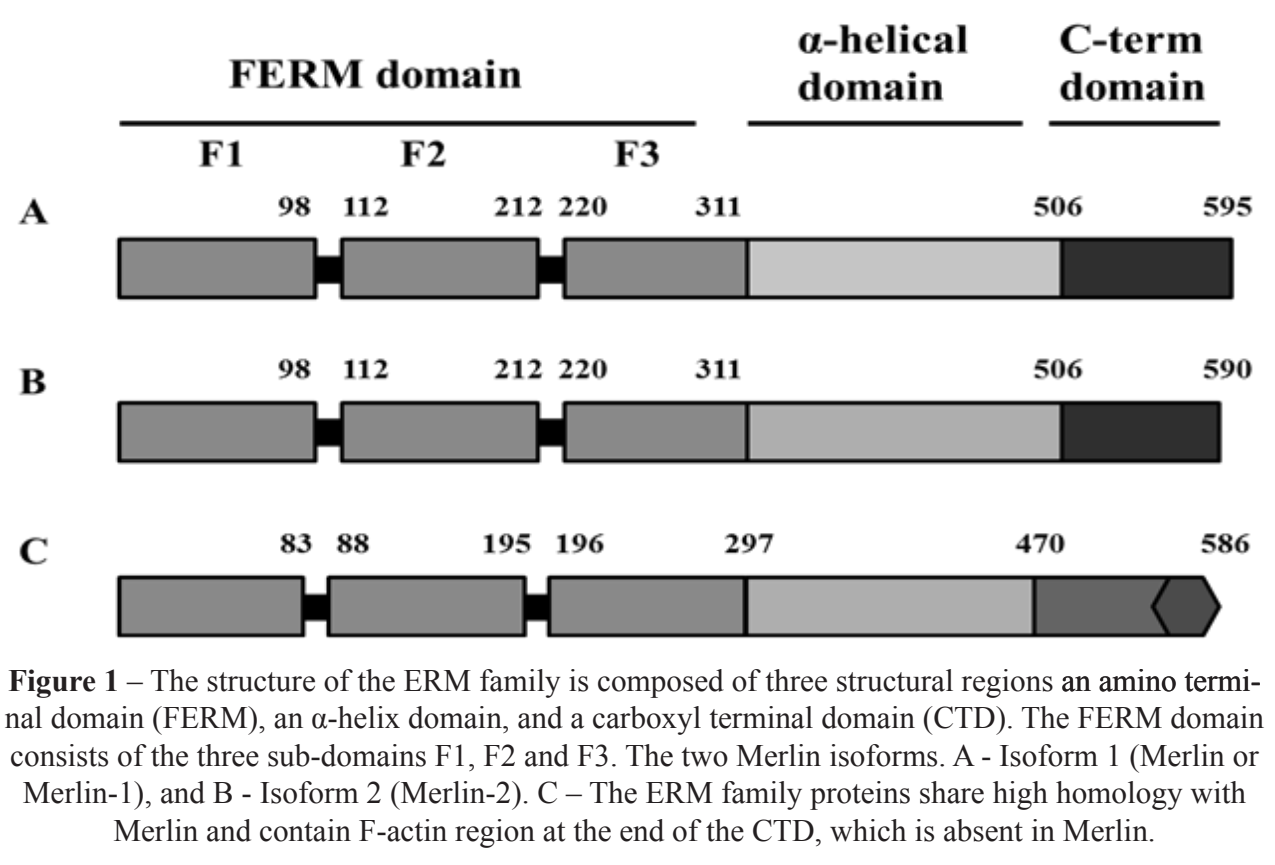

Merlin is localized at the cortex and contact-dependent membrane domains to organize cell junctions [7]. The "Blue box motif", the conserved region corresponding to residues $177-183$ in the F2 subdomain of the FERM domain has been shown to be critical for Merlin growth suppressive function. Merlin's association with actin is not required for growth inhibition, but is necessary for its localization at the plasma membrane in the phosphorylated, so-called dormant state. Loss of Merlin's growth suppressive function results in an impairment of contact-dependent inhibition of proliferation and formation of stable adherens junctions (AJs). It has been shown that Merlin forms an association with adhesion proteins and other AJ components and controls the cadherin-mediated formation of cell-cell adhesions. Cadherins maintain AJs between adjacent cells via interaction with intracellular proteins such as catenins and the actin cyto- skeleton. Thus, the main functions of Merlin include contact inhibition of proliferation and tumor suppression.

\section{Merlin's tumor suppressor activity is regulat- ed by phosphorylation}

The structural transformation of Merlin from an inactive to an active state is regulated by changes in its phosphorylation status. Recent studies have resolved the contradiction between the structure and function of Merlin. Classical studies considered that dephosphorylated Merlin functions as a tumor suppressor in a closed conformation. However, recent studies have shown that phosphorylated or mutated Merlin has a more closed form, leading to impaired growth inhibition, while more open forms have active growth inhibiting activity [13; 15]. Merlin can be inacti- 
vated by phosphorylation at serine 518 by the p21activated protein kinases (PAKs) or by the cAMPdependent protein kinase A (PKA). PAKs belong to a serine/threonine protein kinase family, which are involved in the Rac/Cdc42 signaling pathway. It has been shown that PAK1 phosphorylates Merlin on serine 518 and abolishes its growth suppressor activity [16]. In turn, Merlin can inhibit PAK1 in a contactindependent manner preventing its activation by Rac [17]. In addition, it was shown that cadherin inactivates PAK, which implies contact-independent activation of PAK [18]. Phosphorylation on serine 518 disrupts Merlin association with the actin cytoskeleton, resulting in Merlin intracellular relocalization from the plasma membrane to the cytoplasm [19]. In addition, phosphorylation of Merlin at serine 518 disrupts its interaction with the integral membrane hyaluronan receptor CD44 [20] and hepatocyte growth factor-regulated tyrosine kinase substrate HRS [21], which impairs Merlin's growth suppressive activity. The binding of Merlin to CD44 is associated with hypophosphorylation of Merlin and growth inhibition, while the ERM proteins, competing with Merlin for CD44 binding, prevent the growth suppressive activity of Merlin and contribute to tumorigenesis.

PKA has been shown to phosphorylate Merlin at serine 518 [22]. It has been suggested that phosphorylation of Merlin at serine 518 can convert Merlin into a growth-permissive form through heterodimerization between Merlin and ezrin, while serine 10 phosphorylation plays a role in the cytoskeletal organization and cell motility. Activation of cAMP/ PKA signaling leads to growth proliferation and cell cycle progression in Schwann cells. Conversely, dephosphorylation of serine 518 by MYPT-1-PP1 $\delta$ restores Merlin tumor suppressive function [20; 23].

The serine/threonine kinase AKT, also known as protein kinase $\mathrm{B}(\mathrm{PKB})$, is regulated by phosphorylation at threonine 308 by phosphoinositide-dependent kinase 1 (PDK-1) and is fully activated by additional phosphorylation at serine 473 [24]. AKT has been shown to phosphorylate Merlin at threonine 230 and serine 315, which abolishes Merlin tumor suppressor activity leading to the degradation of Merlin through polyubiquitination [25]. In turn, Merlin suppresses $\mathrm{PI} 3 \mathrm{~K} / \mathrm{AK} T$ signaling through direct binding to PIKEL. Studies have demonstrated that phosphorylation of Merlin on serine 10 by AKT leads to Merlin targeting for ubiquitination by the E3 ubiquitin-protein ligase complex and subsequent proteasome-mediated degradation [26]. This requires multistep phosphorylation of Merlin on serine 318 by PAK or PKA and subsequent phosphorylation on serine 10 by PKA that

Int. j. biol. chem. (Online) leads to a fully open conformation of Merlin resulting in its degradation and ubiquitination. It has been suggested that Merlin phosphorylation on serine 10 by hyperactivated AKT occurs in malignant cells, which leads to Merlin degradation and consequent activation of the PI3K/AKT signaling pathway. Jacob and co-workers have shown that AKT phosphorylation at the serine 308 and threonine 473 sites promoted cell growth and proliferation, indicating activation of the PI3K/AKT signaling pathway in HMS-97 cells and Schwannoma vestibular cells [27]. Although Merlin's tumor suppressor function has been shown to depend on its conformation and phosphorylation status, it is possible that multiple mechanisms may modulate Merlin's growth suppressive activity.

\section{Receptor tyrosine kinases in NF2 signaling}

Receptor tyrosine kinases (RTKs) are implicated in regulation of the Ras/Raf/MEK/ERK and PI3K/ AKT signaling pathways. The ErbB family of receptor tyrosine kinases (RTKs) is a family of transmembrane RTKs, including epidermal growth factor receptor (EGFR), ErbB2/HER2, ErbB3, and ErbB4. RKTs are implicated in cell differentiation and proliferation in Schwann cells and expressed in both vestibular nerve and VS. The EGFR activation starts by binding its specific ligands, such as epidermal growth factor (EGF) and transforming growth factor $\alpha$ (TGF $\alpha)$ to activate Ras/MAPK signaling pathway. Upon ligand binding, RTK inactive monomers activate their cytoplasmic tyrosine kinase domain by dimerization. Merlin inhibits Src binding to ErbB2 and consequently ErbB2-mediated phosphorylation, which prevents downstream mitogenic signaling [28].

Merlin regulates cell-to-cell contact inhibition of proliferation by regulating the accumulation of EGFR, ErbB2, ErbB3, PDGFR and IGF1R growth factors at the plasma membrane in Schwann cells [8; 29]. Activation of ErbB receptors has been linked to increased proliferation, and consequently to the downstream mitogenic signaling [29]. Overexpression of ErbB receptors has been reported in many other human cancers such as human breast and ovarian cancers, as well as transitional cell carcinoma of the bladder [ $30 ; 31]$.

Current study has shown activation of ErbB receptors in human VS and paired normal vestibular nerves using phospho-RTK arrays [32]. Activation and aberrant signaling of ErbB receptors, such as ErbB3 and to a lesser extend activation of EGFR, ErbB2 and ErbB4 have been observed in VS tumors compared to the normal vestibular nerve from the 
same patients. High levels of EGFR phosphorylation have been observed compared to the normal Schwann cells in primary VS culture and human malignant schwannoma HMS-97 cells. In addition, fibroblast growth factor receptor-2 $\alpha$ (FGFR-2 $\alpha$ ), insulin receptor, macrophage-stimulating protein receptor (MSPR), PDGFR- $\beta$, C-RET, and Ephrin type-A4 receptor (EphA4) were upregulated in VS compared to the paired vestibular nerve. Other studies have shown activation of EGFR and ErbB2 in VS and Schwann cells [33; 34]. Lallemand and coworkers have observed activation of ErbB2, ErbB3, insulin-like growth factor 1 receptor (IGF1R), and platelet-derived growth factor receptor- $\beta$ (PDGFR- $\beta$ ) at the plasma membrane in Merlin-deficient confluent but not sub-confluent Schwann cells [29]. The level of EGF was elevated in all NF2-related VS, but not in the sporadic VS, while ErbB ligand was elevated in most of sporadic VS but only in few NF2related VS [33]. Activation of FGFR and PDGFR has been linked to VS growth and progression [35]. The $N F 2$ gene overexpression inhibits schwannoma cell proliferation through promoting PDGFR degradation [36]. Thus, inhibition of RTK aberrant signaling can serve as a potential therapeutic strategy for the treatment of nervous system tumors and other tumors. Therapeutic approaches to the treatment of sporadic and NF2-related VS, as well as other brain tumors will be discussed in the latter part of this review.

\section{Merlin regulates numerous signaling path- ways}

It was previously reported that Merlin is localized on the plasma membrane through the FERM NTD and is associated with microtubules and the cortical actin cytoskeleton [13]. Mutations in the NTD impair the interaction of Merlin with the actincytoskeleton, which leads to its translocation from the plasma membrane into the cytoplasm [37]. It has been generally accepted that changes in Merlin's localization at the plasma membrane disrupts Merlin's suppression of mitogenic signaling and cause the development of NF2 tumors. Later studies have shown that Merlin's tumor suppressor function does not require its association with the actin cytoskeleton $[13 ; 37]$. At the plasma membrane, integrins bind to intracellular matrix components and form a multiprotein complex consisting of RTK, growth factor receptor-bound protein 2 (Grb2), SOS, ERM-actin and Ras [38]. The ERM proteins serve as a scaffold, promoting Ras activation and signaling. Merlin suppresses a variety of signaling pathways by regulat-

Int. j. biol. chem. (Online) ing integrins and tyrosine receptor kinases (RTKs) and the downstream mitogenic signaling of Ras/Raf/ MEK/ERK [20], Rac/PAK/JNK [17; 19], PI3K-Akt [25; 26], mTORC1 [9], FAK-Src [10], Wnt/b-catenin [39] and the EGFR-Ras-ERK pathway [8].

Studies reveal interaction of Merlin with numerous proteins, which can affect Merlin growth suppressor function (Table 1). It has been reported that many putative binding partners interact with Merlin including proteins involved in translation, cell-cycle transit, cytoskeletal organization, and signaling [40]. Merlin regulates cell growth and proliferation through an interaction with proteins of focal adhesion junctions [7], actin [13], $\beta$ II-spectrin [40], microtubules [41], CD44 [20], HRS [21], PIKE-L [42], eIF3c [40], paxillin [43], VprBP [44] (also known as DCAF1), angiomotin [45] and other proteins. As described above, Merlin interacts with several protein kinases, including PAK, PKA, AKT and phosphatase MYPT-1-PP1 $\delta$. The phosphatase MYPT-1-PP1 $\delta$ dephosphorylates Merlin and restores Merlin's antiproliferative function by inhibiting Ras-dependent signaling pathway. The inhibition of MYPT-1-PP1 $\delta$ with the oncoprotein CPI-17 can induce phosphorylation of ERM proteins and Ras activation [20; 23].

Using coimmunoprecipitation, phosphoprotein enrichment, and liquid chromatography coupled with tandem mass spectrometry (LC-MS/MS), several proteins interacting with Merlin have been identified, most of which belong to DNA and chromatin function group, DNA damage response (DDR) proteins, DNA replication and transcription (Davletova et al., unpublished data). Some of these proteins have been reported to be involved in various types of cancer and can serve as both diagnostic and prognostic biomarkers of cancer. Further research is needed to better understand the role of proteins interacting with Merlin in human VS and other types of brain tumors.

In addition to Merlin's localization at the cell cortex and the contact-dependent membrane, Merlin plays a role in the cytoplasm. In the cytoplasm, Merlin associates with actin and tubulin and regulates the dynamics of the cytoskeleton and vesicular transport [13]. Recently, Merlin's tumor suppressor activity has been shown in the nucleus [37]. The FERM domain of Merlin has been reported to be necessary for its nuclear localization. The dephosphorylated form of Merlin, which, as has been demonstrated, has an tumor suppressive activity, is preferentially localized in the nucleus. In the nucleus, Merlin directly interacts with CRL4 ${ }^{\mathrm{DCAF} 1} \mathrm{E} 3$ ubiquitin ligase leading to suppression of oncogenic signaling [37; 45]. Merlin inhibition of CRL4 ${ }^{\mathrm{DCAF}} \mathrm{E} 3$ ubiquitin ligase is linked 
to its tumor suppressor function in contact-inhibited cells. Indeed, mutations abolishing Merlin-DCAF1 binding lead to disruption of nuclear localization and subsequently to tumorigenesis. It has been shown that Merlin's binding to the E3 ubiquitin ligase component DCAF1 has been abolished after serine 10 phosphorylation, resulting in Merlin's targeting to proteasome-mediated degradation [26]. Therefore,
Merlin is involved in various signaling pathway and can play different roles during cell development and tumorigenesis. The mechanisms that regulate Merlin's function as a cell growth suppressor still need to be better understood in order to develop effective therapeutic approaches for the treatment of numerous destructive and debilitating tumors of the nervous system.

Table 1 - Proteins interacting with Merlin and the sites of interaction on Merlin extended to the NTD and/or CTD regions (see references in [40] if not shown otherwise; ND - not identified)

\begin{tabular}{|c|c|c|c|}
\hline Protein & NTD & Protein & CTD \\
\hline \multicolumn{4}{|c|}{ Protein kinases/phosphotases affecting Merlin suppressive actiivity } \\
\hline PAK1 $[16 ; 17]$ & $1-313$ & & \\
\hline PKA [22] & ND & & \\
\hline MYPT-1-PP1 $\delta[23]$ & $312-341$ & & \\
\hline \multicolumn{4}{|c|}{ Structural proteins supporting Merlin suppressive activity } \\
\hline Microtubules & $122-302$ & $\beta I I-$ spectrin & $469-590$ \\
\hline Actin & $178-367$ & & \\
\hline \multicolumn{4}{|c|}{ Growth activators suppressed by Merlin } \\
\hline CD44 & $1-50$ & TRBP & $288-595$ \\
\hline RalGDS & $1-228$ & HEI10 & $306-339$ \\
\hline eIF3c & $1-304$ & EG1/Magicin & $340-590 / 595$ \\
\hline Ezrin & $1-313$ & Syntetin & $566-595$ \\
\hline Radixin & ND & Angiomotin [45] & ND \\
\hline Moezin & $1-332$ & & \\
\hline Layilin & $1-332$ & & \\
\hline PIKE-L [42] & $1-332$ & & \\
\hline NHERF & $1-332$ & & \\
\hline N-WASP & $1-332$ & & \\
\hline RhoGDI & $1-341$ & & \\
\hline VprBP [44] & ND & & \\
\hline \multicolumn{4}{|c|}{ Growth inhibitors supporting Merlin suppressive activity } \\
\hline RhoGDI & $1-341$ & HRS [21] & $453-557$ \\
\hline NGB & $1-52 ; 288-344$ & & \\
\hline DCC & $1-341$ & & \\
\hline MAP & $288-595$ & & \\
\hline Paxillin [43] & $50-70 ; 425-450$ & & \\
\hline \multicolumn{4}{|c|}{ Proteins interacting with Merlin with unclear effect } \\
\hline Myelination & $1-314$ & $\mathrm{RI} \beta$ & $463-480$ \\
\hline SCHIP-1 & $1-27 ; 280-323$ & CRM1/exportin & $539-551$ \\
\hline
\end{tabular}




\section{Therapeutic strategies for the treatment of VS and other brain tumors}

Currently, there are no medical therapies available for the treatment of NF2-related and sporadic VS and other nervous system tumors. The current standard of treatment remains local tumor control by surgical excision and stereotactic radiation, which can often cause complications from damage to nervous and CNS structures [4]. Moreover, radiation treatment can lead to malignant transformation and elevated growth of tumors [46]. Merlin plays a role as a cell growth suppressor and interacts with a number of proteins involved in various signaling pathways. Deregulation of one of the components in these signaling pathways can cause tumor formation. Targeted small molecule inhibitors may serve as potent therapeutic agents for the treatment of these tumours.

The PI3K/AKT signaling pathway is deregulated in VS and other tumors. A novel celecoxib-derivative OSU-03012 (developed by Dr. C.-S. Chen at Ohio State University, also called AR-12) lacking cyclooxygenase (COX2) inhibitory activity has been tested in several types of cancer cells [24] and is currently undergoing Phase I clinical trial as an anticancer agent in patients with solid and liquid tumors. The mechanism of OSU-03012 is partly modulated via direct inhibition of 3-phosphoinositide-dependent protein kinase-1 (PDK-1) within the PI3K/AKT pathway. Another anticancer mechanism of OSU-03012 involves endoplasmic reticulum (ER) stress signaling via activation of PKR-like ER kinases (PERK), downregulation of HSP70 family chaperone GRP78/ BiP/HSPA5, and a caspase-independent, cathepsindependent autophagy-dependent form of cell death [47]. It has been shown that activation and phosphorylation of AKT has been linked to cell proliferation and VS tumor formation [48]. A dose-dependent inhibition of cell proliferation and apoptosis induced by OSU-03012 was observed both in benign human VS and malignant schwannoma cells, as well as mouse Nf2-deficient Schwann and schwannoma cells. OSU03012 potent anti-tumor activity was demonstrated in malignant schwannoma xenografts in SCID mice.

Current study revealed aberrant activation of ErbB family RTKs in human VS [32]. The treatment with EGFR inhibitor Erlotinib and EGFR/ErbB2 inhibitor Lapatinib inhibited cell proliferation in VS and schwannoma cells. Erlotinib treatment was more potent in growth inhibition compared to Lapatinib and resulted in a decreased level of phospho-ErbB receptors including EGFR in primary VS, as well as ErbB3, and ErbB4 in human malignant schwannoma

Int. j. biol. chem. (Online) cells. Although Lapatinib was not very potent in inhibiting cell growth, it is possible that the combined action of this inhibitor with OSU-03012 might provide a better outcome and is currently being tested. Several studies have demonstrated the application of RTK inhibitors as an effective therapeutic approach for the treatment of human VS and other nervous system tumors $[33 ; 34]$. The treatment with OSU03012 and the inhibitor of ERBB1/2/4, Lapatinib, or the HSP90 inhibitor 17-N-Allylamino-17-demethoxygeldanamycin (17AAG) reduced expression of ERBB1/2/4 growth factor receptors in glioblastoma and medulloblastoma cells [47]. Therefore, targeting ERBB growth factor receptors could reduce cell proliferation through inhibition of ERK1/2 and PI3K/ AKT signaling pathways.

Preclinical and clinical studies of the potential therapeutic targets, such as lapatinib, everolimus, erlotinib and bevacizumab have been reported for the treatment of VS, NF2 and other brain tumors [49-51]. Other potential targets for therapeutic strategies include inhibitors of small GTP binding proteins, and GTPases that can inhibit Ras, Rac, and MAPK signaling pathways and have been used for certain types of cancer. Several PAK inhibitors have been tested and may be useful for treating VS and NF2 related tumors [52; 53]. The inhibition of mTORC1 signaling had a direct inhibitory effect on VS growth. The Phase II study of mTORC1 inhibition by everolimus has shown delayed growth of NF2-schwannomas [54]. A better understanding of deregulated signal transduction pathways is crucial in exploring therapeutic options and preclinical testing of effective chemical agents for treating tumors of the nervous system.

\section{Conclusion}

Merlin negatively regulates cell growth and proliferation and is involved in multiple signaling pathways. It has been shown aberrant activation of ErbB family receptor tyrosine kinases (RTK) in human VS. Current study has shown that inhibition of receptor tyrosine kinases can serve as an alternative approach for the treatment of sporadic and NF2related VS. Other potential targets for therapeutic strategies include chemical agents that can inhibit deregulated mitogenic pathways such as Ras, Rac, and MAPK signaling pathways. A better understanding of the mechanisms underlying VS tumorigenesis can help to develop novel potent, effective and nontoxic chemical agents to supress tumor growth and metastasis. Several preclinical and clinical trials are 
underway as the potential therapeutic targets for the treatment of VS and NF2-related tumors.

\section{Acknowledgment}

I wish to express my sincere thanks to Drs. D. Bradley Welling and Long-Sheng Chang for their mentorship and financial support from the Department of Otolaryngology-Head and Neck Surgery, Ohio State University and the Center for Childhood Cancer, the Research Institute at Nationwide Children's Hospital, Columbus, Ohio, USA.

\section{References}

1. Evans D.G., Sainio M., Baser M. E. (2000) Neurofibromatosis type 2. J. Med. Genet., vol. 37, pp. 897-904.

2. Rouleau G.A., Merel P., Lutchman M., Sanson M., Zucman J. et al. (1993). Alteration in a new gene encoding a putative membrane organizing protein causes neuro-fibromatosis type 2. Nature, vol. 363, pp. 515-521.

3. Evans D.G., Huson S.M., Donnai D., Neary W., Blair V. et al. (1992) A genetic study of type 2 neurofibromatosis in the United Kingdom. I. Prevalence, mutation rate, fitness, and confirmation of maternal transmission effect on severity. J Med Genet., vol. 29 , pp. 841-846.

4. Gutmann D.H., Giovannini M. (2002) Mouse models of neurofibromatosis 1 and 2. Neoplasia, vol. 4, no. 4, pp. 279-290.

5. Larsson J., Ohishi M., Garrison B., Aspling M., Janzen V. et al. (2008) Nf2/merlin regulates hematopoietic stem cell behavior by altering microenvironmental architecture. Cell Stem Cell, vol. 3, pp. 221-227.

6. Benhamouche S., Curto M., Saotome I., Gladden A.B., Liu C.-H. et al. (2010) Nf2/merlin controls progenitor homeostasis and tumorigenesis in the liver. Genes Dev., vol. 24, pp. 1718-1730.

7. Lallemand D., Curto M, Saotome I., Giovannini M., McClatchey A.I. (2003) NF2-deficiency promotes tumorigenesis and metastasis by destabilizing adherens junctions. Genes Dev., vol. 7, pp. 10901100.

8. Curto M., Cole B. K., Lallemand D., Liu C., McClatchey A. I. (2007) Contact-dependent inhibition of EGFR signaling by Nf2/merlin. $J$ Cell Biol., vol. 177 , pp. 893-903.

9. James M.F., Han S., Polizzano C., Plotkin S.R., Manning B.D. et al. (2009) NF2/Merlin is a novel negative regulator of mTOR complex 1 , and activa-

Int. j. biol. chem. (Online) tion of mTORC1 is associated with meningioma and schwannoma growth. Mol and Cell Biol., vol. 29, no. 15, pp. 4250-4261.

10. Poulikakos P. I., Xiao G. H., Gallagher R., Jablonski S., Jhanwar S. C. et al. (2006) Re-expression of the tumor suppressor NF2/Merlin inhibits invasiveness in mesothelioma cells and negatively regulates FAK. Oncogene, vol. 25, pp. 5960-5968.

11. Guerrero P.A., Yin W., Camacho L., Marchetti D. (2015) Oncogenic Role of Merlin/NF2 in Glioblastoma. Oncogene, vol. 34, no. 20, pp. 26212630.

12. Morrow K.A., Das S., Metge B.J., Ye K., Mulekar M.S. et al. (2011) Loss of tumor suppressor Merlin in advanced breast cancer is due to posttranslational regulation. J Biol Chem., vol. 286, pp. 40376-40385.

13. Lallemand D., Saint-Amaux A.L., Giovannini M. (2009a) Tumor-suppression functions of merlin are independent of its role as an organizer of the actin cytoskeleton in Schwann cells. J Cell Sci., vol. 122, pp. 4141-4149.

14. Gutmann D.H., Geist R.T., Xu H., Kim J.S., Saporito-Irwin S. (1998) Defects in neurofibromatosis 2 protein function can arise at multiple levels. Hum Mol Genet., vol. 7, no. 3, pp. 335-345.

15. Sher I., Hanemann C.O., Karplus P.A., Bretscher A. (2012) The tumor suppressor merlin controls growth in its open state, and phosphorylation converts it to a less-active more-closed state. Dev Cell., vol. 22, pp. 703-705.

16. Kissil J.L., Johnson K.C., Eckman M.S., Jacks T. (2002). Merlin phosphorylation by p21-activated kinase 2 and effects of phosphorylation on merlin localization. J Biol Chem., vol. 277, pp. 10394-10399.

17. Kissil J.L., Wilker E.W., Johnson K.C., Eckman M.S., Yaffe M.B. et al. (2003). Merlin, the product of the Nf2 tumor suppressor gene, is an inhibitor of the p21-activated kinase, Pak1. Mol Cell., vol. 12, pp. 841-849.

18. Okada T., Lopez-Lago M., Giancotti F. G. (2005) Merlin/NF-2 mediates contact inhibition of growth by suppressing recruitment of Rac to the plasma membrane. J Cell Biol., vol. 171, pp. 361-371.

19. Shaw R.J., Paez J.G., Curto M., Yaktine A., Pruitt W.M. et al. (2001) The Nf2 tumor suppressor, merlin, functions in Rac-dependent signaling. Dev Cell., vol. 1, pp. 63-72.

20. Morrison H., Sherman L.S., Legg J., Banine F., Isacke C. et al. (2001) The NF2 tumor suppressor gene product, merlin, mediates contact inhibition of growth through interactions with CD44. Genes Dev., vol. 15 , pp. $968-980$. 
21. Scoles D.R., Huynh D.P., Chen M.S., Burke S.P., Gutmann D.H. et al. (2000) The neurofibromatosis 2 tumor suppressor protein interacts with hepatocyte growth factor-regulated tyrosine kinase substrate. Hum Mol Genet., vol. 9, no. 11, pp. 15671574.

22. Alfthan K., Heiska L., Grönholm M., Renkema G.H., Carpén O. (2004). Cyclic AMP-dependent protein kinase phosphorylates merlin at serine 518 independently of p21-activated kinase and promotes merlin-ezrin heterodimerization. J Biol Chem., vol. 279, pp. 18559-18566.

23. Jin H., Sperka T., Herrlich P., Morrison H. (2006) Tumorigenic transformation by CPI-17 through inhibition of a merlin phosphatase, Nature, vol. 442 , pp. 576-579.

24. Zhu J., Huang J.W., Tseng P.H., Yang Y.T., Fowble J. et al. (2004) From the cyclooxygenase-2 inhibitor celecoxib to a novel class of 3-phosphoinositide-dependent protein kinase-1 inhibitors. Cancer Res., vol. 64, pp. 4309-4318.

25. Okada M., Wang Y., Jang S.-W., Tang X., Neri L.M. et al. (2009) Akt Phosphorylation of Merlin Enhances Its Binding to Phosphatidylinositols and Inhibits the Tumor-Suppressive Activities of Merlin. Cancer Res., vol. 69, no. 9, pp. 4043-4051.

26. Laulajainen M., Muranen T., Nyman T.A., Carpen O., Gronholm M. (2011) Multistep phosphorylation by oncogenic kinases enhances the degradation of the NF2 tumor suppressor Merlin. Neoplasia, vol. 13, pp. 643-652.

27. Jacob A., Lee T.X., Neff B.A., Miller S., Welling B. et al. (2008) Phosphatidylinositol 3-kinase/AKT pathway activation in human vestibular schwannoma. Otol Neurotol., vol. 29, pp. 58-68.

28. Houshmandi S.S., Emnett R.J., Giovannini M., Gutmann D.H. (2009) The Neurofibromatosis 2 Protein, Merlin, Regulates Glial Cell Growth in an ErbB2- and Src-Dependent Manner. Mol and Cell Biol., vol. 29, no. 6, pp. 1472-1486.

29. Lallemand D., Manent J., Couvelard A., Watilliaux A., Siena M. et al. (2009b) Merlin regulates transmembrane receptor accumulation and signaling at the plasma membrane in primary mouse Schwann cells and in human schwannomas. Oncogene, vol. 28, pp. 854-865.

30. Bieche I., Onody P., Tozlu S., Driouch K., Vidaud M. et al. (2003) Prognostic value of ERBB family mRNA expression in breast carcinomas. Int $\mathrm{J}$ Cancer, vol. 106, pp. 758-765.

31. Junttila T.T., Laato M., Vablberg T., Soderstrom K.O., Visacorpi T. et al. (2003) Identification of patients with transitional cell carcinoma of the

Int. j. biol. chem. (Online) bladder overexpressing ErbB2, ErbB3, or specific ErbB4 isoforms: real-time reverse transcription-PCR analysis in estimation of ErbB receptor status from cancer patients. Clin Cancer Res., vol. 9, pp. 53465357.

32. Bush M. L., Burns S. S., Oblinger J., Davletova S., Chang L.-S. et al. (2012) Treatment of Vestibular Schwannoma Cells with ErbB Inhibitors. Otol. Neurotol., vol. 33, no. 2, pp. 244-257.

33. Doherty J.K., Ongkeko W., Crawley B., Andalibi A., Ryan A.F. et al. (2008) ErbB and Nrg: potential molecular targets for vestibular schwannoma pharmacotherapy. Otol Neurotol., vol. 29, pp. 50-57.

34. Ammoun S., Cunliffe C.H., Allen J.C., Chiriboga L., Giancotti F.G. et al. (2010) ErbB/HER receptor activation and preclinical efficacy of lapatinib in vestibular schwannoma. Neuro Oncol., vol. 12, pp. 834-843.

35. Ammoun S., Flaiz C., Ristic N., Schuldt J., Hanemann C.O. (2008) Dissecting and targeting the growth factor-dependent and growth factor-independent extracellular signal-regulated kinase pathway in human schwannoma. Cancer Res., vol. 68, pp. 5236-5245.

36. Fraenzer J.T., Pan H., Minimo Jr. L., Smith G.M., Knauer D. et al. (2003). Overexpression of the NF2 gene inhibits schwannoma cell proliferation through promoting PDGFR degradation. Int J Oncol., vol. 23, pp. 1493-1500.

37. Cooper J., Giancotti F.G. (2014) Molecular insights into NF2/Merlin tumor suppressor function. FEBS Lett., vol. 588, no. 16, pp. 2743-52.

38. Sperka T., Geißler K.J., Merkel U., Scholl I., Rubio I. et al. (2011) Activation of Ras Requires the ERM-Dependent Link of Actin to the Plasma Membrane. PLoS One, vol. 6, no. 11, e27511.

39. Bosco E.E., Nakai Y., Hennigan R.F., Ratner N., Zheng Y. (2010) NF2-deficient cells depend on the Rac1-canonical Wnt signaling pathway to promote the loss of contact inhibition of proliferation. Oncogene, vol. 29, pp. 2540-2549.

40. Scoles D.R. (2008) The merlin interacting proteins reveal multiple targets for NF2 therapy. Biochim Biophys Acta, vol. 1785, no. 1, pp. 32-54.

41. Muranen T., Grönholm M., Lampin A., Lallemand D., Zhao F. et al. (2007) The tumor suppressor merlin interacts with microtubules and modulates Schwann cell microtubule cytoskeleton. Hum Mol Genet., vol. 16, no. 14, pp. 1742-1751.

42. Rong R., Surace E.I., Haipek C.A., Gutmann D.H., Ye K. (2004). Serine 518 phosphorylation modulates merlin intramolecular association and binding to critical effectors important for NF2 growth suppression. Oncogene, vol. 23, pp. 8447-8454. 
43. Fernandez-Valle C., Tang Y., Ricard J., Rodenas-Ruano A., Taylor A. et al. (2002) Paxillin binds schwannomin and regulates its density-dependent localization and effect on cell morphology. Nat. Genet., vol. 31, pp. 354-362.

44. Huang J., Chen J. (2008) VprBP targets Merlin to the Roc1-Cul4A-DDB1 E3 ligase complex for degradation. Oncogene, vol. 27, pp. 4056-4064.

45. Yi C., Troutman S., Fera D., Stemmer-Rachamimov A., Avila J.L. (2011) A tight junction-associated Merlin-angiomotin complex mediates Merlin's regulation of mitogenic signaling and tumor suppressive functions. Cancer Cell, vol. 19, pp. 527-40.

46. Balasubramaniam A., Shannon P., Hodaie M., Laperriere N., Michaels H. et al. (2007) Glioblastoma multiforme after stereotactic radiotherapy for acoustic neuroma: case report and review of the literature. Neuro-Oncol., vol. 9, pp. 447-453.

47. Booth L., Cazanave S.C., Hamed H.A., Yacoub A., Ogretmen B. et al. (2012b) OSU-03012 suppresses GRP78/BiP expression that causes PERK-dependent increases in tumor cell killing. Cancer Biol Ther., vol. 13, pp. 224-236.

48. Lee T.X., Packer M.D., Huang J., Akhmametyeva E.M., Kulp C.D. et al. (2009) Growth Inhibitory and Anti-Tumour Activities of OSU-03012, a Novel PDK-1 Inhibitor, on Vestibular Schwannoma and Malignant Schwannoma cells. Eur J Cancer, vol. 45, no. 9, pp. 1709-1720.

49. Karajannis M.A., Legault G., Hagiwara M., Ballas M.S., Brown K. et al. (2012) Phase II trial of lapatinib in adult and pediatric patients with neurofi- bromatosis type 2 and progressive vestibular schwannomas. Neuro Oncol., vol. 14, no. 9, pp. 1163-1170.

50. Karajannis M.A., Legault G., Hagiwara M., Giancotti F.G., Filatov A. et al. (2014) Phase II study of everolimus in children and adults with neurofibromatosis type 2 and progressive vestibular schwannomas. Neuro Oncol., vol. 16, no. 2, pp. 292297.

51. Ouerdani A., Goutagny S., Kalamarides M., Trocóniz I.F., Ribba B. (2016) Mechanism-based modeling of the clinical effects of bevacizumab and everolimus on vestibular schwannomas of patients with neurofibromatosis type 2. Cancer Chem Pharmacol., vol. 77, no. 6, pp. 1263-1273.

52. Licciulli S., Maksimoska J., Zhou C., Troutman S., Kota S. et al. (2013) FRAX597, a Small Molecule Inhibitor of the p21-activated Kinases, Inhibits Tumorigenesis of Neurofibromatosis Type 2 (NF2)associated Schwannomas. J Biol Chem., vol. 288, no. 40, pp. 29105-29114.

53. Mercado-Pimentel M.E., Miller C., Rolph D.N., Villalobos E.F., Dunn A.M. et al. (2017) Inhibiting p21-Activated Kinase Induces Cell Death in Vestibular Schwannoma and Meningioma via Mitotic Catastrophe. Otol Neurotol., vol. 38, no. 1, pp. 139-146.

54. Goutagny S., Raymond E., Esposito-Farese M., Trunet S., Mawrin C. et al. (2015) Phase II study of mTORC1 inhibition by everolimus in neurofibromatosis type 2 patients with growing vestibular schwannomas. J Neurooncol., vol. 122, no. 2, pp. 313-320. 